\section{Estrutura dimensional da versão brasileira do Functional Activities Questionnaire (FAQ-BR)}

\author{
Dimensional structure of the Brazilian version of \\ the Functional Activities Questionnaire (FAQ-BR)
}

\author{
Estructura dimensional de la versión brasileña \\ del Functional Activities Questionnaire (FAQ-BR)
}

Rafael Tavares Jomar 1

Roberto Alves Lourenço 1

Claudia de Souza Lopes 1

doi: 10.1590/0102-311X00209917

\section{Resumo}

Este estudo teve por objetivo avaliar a estrutura dimensional e a consistência interna da versão brasileira do Functional Activities Questionnaire (FAQ-BR) para aferição da capacidade funcional do idoso, com base no relato de informante. Trata-se de um estudo seccional desenvolvido com 525 informantes de idosos não institucionalizados, em que análises fatoriais confirmatórias $(A F C)$ e análises fatoriais exploratórias $(A F C / E)$, modeladas segundo os princípios da AFC, foram usadas para identificar o modelo mais parcimonioso e mais bem ajustado. A consistência interna do FAQ-BR foi avaliada pela confiabilidade composta, e correlações entre suas dimensões foram examinadas a fim de investigar validade fatorial discriminante. A AFC não corroborou a estrutura unidimensional originalmente proposta. Em seguida, a AFC/E ajustou uma estrutura bidimensional que foi reavaliada por AFC, evidenciando um modelo sem cargas cruzadas como o mais parcimonioso $e$ com adequados índices de ajuste. A consistência interna do FAQ-BR foi considerada satisfatória, e a correlação entre suas dimensões, aceitável. Esses achados são importantes, pois revelam a capacidade de a escala capturar o construto capacidade funcional do idoso em um contexto sociocultural distinto daquele em que foi desenvolvido. Portanto, o FAQ-BR pode ser considerado um instrumento bidimensional apropriado para aferir a capacidade funcional do idoso com base no relato do informante, e seu uso em pesquisas com populações semelhantes é recomendado.

Avaliação de Programas e Instrumentos de Pesquisa; Análise Fatorial; Estudos de Validação; Atividades Cotidianas; Saúde do Idoso

\author{
Correspondência \\ R. T. Jomar \\ Universidade do Estado do Rio de Janeiro. \\ Rua São Francisco Xavier 524, Bloco D, 7o andar, \\ Rio de Janeiro, RJ 20550-013, Brasil. \\ rafaeljomar@yahoo.com.br \\ 1 Universidade do Estado do Rio de Janeiro, Rio de Janeiro, \\ Brasil.
}




\section{Introdução}

No idoso, a incapacidade funcional é definida pela necessidade de assistência parcial ou total de outrem para a realização de uma atividade de vida diária ou pela não capacidade de realizá-la. Em outras palavras, é a inaptidão do idoso para manter as habilidades físicas e mentais necessárias a uma vida independente e autônoma 1 . Opostamente, a capacidade funcional é uma medida de independência, um importante indicador do envelhecimento ativo, que se refere à potencialidade para realizar determinado ato sem necessidade de ajuda ou à plena realização de uma tarefa ou ação desempenhada pelo idoso 2 .

Habitualmente, a aferição da capacidade funcional do idoso é realizada por meio da aplicação de questionários baseados no autorrelato e sistematizados por meio de escalas, destacando-se aquelas que avaliam o desempenho em atividades básicas e instrumentais de vida diária 3,4. Entretanto, outra abordagem pode ser utilizada para aferir a capacidade funcional do idoso: a entrevista com um informante. Relatos de um informante que esteja familiarizado com o desempenho do idoso em atividades de vida diária podem auxiliar o estabelecimento do diagnóstico de incapacidade funcional. Em geral, os testemunhos dessas pessoas são confiáveis, pois são capazes de descrever retrospectiva e adequadamente os sintomas anormais apresentados pelo idoso, já que conhecem sua rotina, seu estado de desempenho funcional e suas limitações inerentes à dependência 5,6.

O Functional Activities Questionnaire (FAQ) 7, baseado no relato do informante, é a escala de aferição da capacidade funcional do idoso mais utilizada em pesquisas $4,8,9$. O questionário se propõe a aferir o grau de dependência no desempenho de diversas atividades instrumentais de vida diária e foi desenvolvido em língua inglesa por um grupo de pesquisadores do Departamento de Neurologia da Universidade da Califórnia (Estados Unidos) para ser aplicado ao cônjuge, parente ou amigo próximo do idoso. Assim, seus resultados são baseados no relato de um informante que conhece a rotina e o desempenho do idoso em atividades de vida diária 7.

O estudo que descreve o desenvolvimento e a validação do FAQ, originalmente proposto como escala unidimensional, foi conduzido com 195 informantes de idosos não institucionalizados e reportou elevados valores de confiabilidade interexaminador (r), que variaram de 0,80 a 0,97, e de correlação item-total $(\mathrm{r}=0,80)$. Além disso, apontou adequada validade de critério preditiva de demência diagnosticada por médico neurologista (sensibilidade de $85 \%$ e especificidade de $81 \%$ ), quando o informante assinalou dependência do idoso em mais de duas atividades instrumentais de vida diária 7 .

O FAQ possui dez itens com seis opções de resposta: quando a resposta do informante a um item aponta independência do idoso na execução de uma atividade, pontua-se 0; caso aponte para a dificuldade na execução dela, pontua-se 1; quando aponta necessidade de ajuda para desempenhá-la, pontua-se 2; e quando não consegue executá-la, pontua-se 3. Para aquelas atividades que, habitualmente, não eram realizadas pelo idoso, o informante precisa especificar se o idoso seria capaz (0 ponto) ou não (1 ponto) de realizá-la, se fosse necessário. A pontuação mínima da escala é 0, e a máxima é 30. Logo, quanto menor for sua pontuação global, maior o nível de independência na execução de atividades instrumentais de vida diária 7 .

Estudo de revisão sobre o uso do FAQ no Brasil 8 aponta que versões em português dessa escala, não submetidas a um processo formal de adaptação transcultural para o contexto brasileiro, têm sido muito utilizadas. Embora tais versões tenham características semelhantes à original, sua estrutura foi alterada, e investigações que avaliassem suas qualidades psicométricas não estavam disponíveis até que um estudo 10 foi conduzido com esse fim. Cabe destacar, entretanto, que tal estudo, além de avaliar as propriedades psicométricas de uma versão do FAQ oriunda de um processo de tradução desconhecido, avaliou seu comportamento psicométrico entre idosos 10, e não entre seus informantes, para quem sua aplicação foi originalmente proposta 7 .

No processo de validação de instrumentos de aferição, a questão dimensional não é apenas importante, mas fundamental, pois a estrutura dimensional indica a forma como o instrumento deve ser concretamente operacionalizado. A utilização de um escore global somente se justifica se uma escala é efetivamente unidimensional. Caso contrário, usar subescalas separadamente pode ser a melhor forma de acomodar diferentes dimensões de um construto. Além disso, diferentes formas de operacionalização de uma escala implicam diferentes resultados que, em última instância, prejudicam comparações entre estudos 11 . 
Diante da inexistência de uma escala validada no Brasil para aferição da capacidade funcional do idoso, baseada no relato do informante, Sanchez et al. 12 conduziram as primeiras etapas da adaptação transcultural do FAQ, a saber: equivalências conceitual, de itens, semântica, operacional e avaliação de consistência interna e confiabilidade. A versão brasileira do FAQ - FAQ-BR (Quadro 1), aplicada ao informante, apresentou consistência interna de 0,95 (alfa de Cronbach) e coeficiente de correlação intraclasse de 0,9712.

Considerando a importância da avaliação das propriedades de mensuração de instrumentos de medida adaptados a novos contextos culturais e que o processo de adaptação transcultural do FAQ para o contexto brasileiro deve continuar seguindo passos metodológicos sólidos a fim de atingir a equivalência funcional e, desse modo, ser utilizado em pesquisas, este estudo teve por objetivo avaliar a estrutura dimensional e a consistência interna da FAQ-BR 12 para aferição da capacidade funcional do idoso, com base no relato do informante.

\section{Métodos}

Estudo seccional desenvolvido na linha de base de uma coorte de idosos não institucionalizados, de idade maior ou igual a 65 anos, clientes de uma operadora de saúde: Fragilidade em Idosos Brasileiros seção Rio de Janeiro (FIBRA-RJ). O principal objetivo foi investigar as características e os fatores de risco de natureza biológica e ambiental da síndrome de fragilidade em idosos. A condução do FIBRA-RJ foi aprovada pelo Comitê de Ética em Pesquisa do Hospital Universitário Pedro Ernesto da Universidade do Estado do Rio de Janeiro (1.850/2007), e seus aspectos metodológicos foram apresentados detalhadamente em outra publicação 13 .

A população do presente estudo foi constituída por informantes de 525 idosos participantes da linha de base do FIBRA-RJ que, por sua vez, obtiveram pontuação menor ou igual a 27 no Mini-Exame do Estado Mental 14 e maior ou igual a 5 no FAQ-BR 12. A escala sob investigação foi aplicada por entrevistadores devidamente treinados por meio de contato telefônico, com duração média de sete minutos, ao informante que conhecia a rotina e o desempenho do idoso em atividades de vida diária.

Com o intuito de avaliar a estrutura unidimensional do FAQ-BR, conforme proposta em sua versão original, análise fatorial confirmatória (AFC) foi conduzida, empregando-se o estimador Weighted Least Squares Mean and Variance Adjusted (WLSMV) e matrizes de correlações policóricas, adequados para itens com opções de resposta ordinais 15 , como as da escala sob investigação. Cargas fatoriais $(\lambda)$ $>0,40$ foram consideradas satisfatórias, e erros de mensuração $(\delta) \geq 0,70$, altos e inadequados 15 . A qualidade do ajuste do modelo foi avaliada por meio dos índices de ajuste incremental Comparative Fit Index (CFI) > 0,90 e Tuckey-Lewis Index (TLI) > 0,90 e do Root Mean Square Error of Aproximation (RMSEA) < 0,08 16. Considerou-se, ainda, o intervalo de 90\% de confiança (IC90\%) do RMSEA, que ofereceu suporte adicional para avaliar o ajuste do modelo (limite superior $<0,08$ ) 15 .

Antecipando um possível mau ajuste do modelo original e prevendo estruturas alternativas, a estrutura dimensional do FAQ-BR foi reavaliada por meio de modelos de equação estrutural exploratórios. Esses modelos aumentam a capacidade das análises fatoriais exploratórias (AFE) tradicionais, permitindo a avaliação de correlações residuais, cuja presença indica possíveis redundâncias de itens 15 . Tal procedimento consiste em ajustar uma sequência de modelos exploratórios seguindo métodos empregados em análises fatoriais confirmatórias (AFC/E) que, por sua vez, permite uma rotação. Nesta análise, foi utilizada a rotação oblíqua Geomin 17. Correlações entre as dimensões do FAQ-BR foram avaliadas, considerando-se valores $>0,85$ como sugestivos de falta de validade fatorial discriminante 15 .

Na sequência, o melhor modelo identificado pela AFC/E foi reavaliado por meio de AFC estrita, empregando-se o estimador WLSMV, matrizes de correlações policóricas 15 e os índices de ajuste CFI, TLI e RMSEA 16. Além de reavaliar cargas fatoriais e erros de mensuração, este procedimento envolveu a avaliação da consistência interna por meio da confiabilidade composta (CC), visto que o alfa de Cronbach subestima a confiabilidade em situações de violação de tal equivalência, em que todos os itens deveriam ter as mesmas cargas fatoriais, mesmo que suas variâncias únicas sejam diferentes. A $C C$ varia de 0 a 1 , sendo considerada satisfatória quando $\geq 0,70$ 15. Correlações entre as dimensões do FAQ-BR também foram avaliadas nesta etapa 15. 
Quadro 1

Versão brasileira do Functional Activities Questionnaire (FAQ-BR).

\begin{tabular}{|c|c|c|c|}
\hline $\begin{array}{c}\text { A. Preenche cheques, paga contas, verifica o saldo no talão de } \\
\text { cheque, controla as necessidades financeiras? }\end{array}$ & $\begin{array}{c}\text { F. Prepara uma refeição completa (p.ex.: carne, frango ou peixe, } \\
\text { legumes, sobremesa)? }\end{array}$ \\
\hline $\begin{array}{c}\text { Atualmente alguém passou a fazer alguma ou todas essas atividades } \\
\text { pelo idoso. }\end{array}$ & $\begin{array}{c}\text { Atualmente alguém passou a fazer essas atividades pelo idoso. } \\
\text { Precisa ser lembrado ou requer ajuda de outras pessoas. }\end{array}$ & Precisa de ajuda ou tem problemas frequentes. \\
\hline $\begin{array}{c}\text { Realiza as atividades sem nenhum lembrete ou auxílio, porém com mais } \\
\text { dificuldade ou com pior resultado. }\end{array}$ & Realiza a atividade sem nenhuma ajuda. \\
\hline Nunca fez e teria dificuldade para começar a fazer agora. & Nunca fez e teria dificuldade para começar a fazer agora, mesmo dificuldade do que antes. \\
\hline com treinamento.
\end{tabular}

treinamento, se fosse necessário.

B. Faz seguro (de vida, de carro, de casa), lida com negócios ou documentos, faz imposto de renda?

Atualmente alguém que não fazia passou a fazer essas atividades pelo idoso sempre ou quase sempre.

Precisa ser lembrado com mais frequência ou necessita de mais ajuda de outras pessoas do que no passado.

Realiza as atividades sem lembretes ou ajuda, porém com mais dificuldade ou com pior resultado do que no passado.

Realiza as atividades sem nenhum lembrete ou ajuda.

Nunca fez e teria dificuldade para começar a fazer agora, mesmo com treinamento.

Não fazia regularmente, mas poderia fazer agora, se fosse necessário.

C. Compra roupas, utilidades domésticas e artigos de mercearia sozinho(a)?

Atualmente alguém passou a fazer alguma ou todas essas atividades

pelo idoso.

G. Acompanha os eventos atuais no bairro ou nacionalmente?

Realiza as atividades sem nenhum lembrete ou auxílio, porém com mais dificuldade ou pior resultado.

Realiza as atividades sem nenhum lembrete ou ajuda.

Nunca fez e teria dificuldade para começar a fazer agora.

Não fazia regularmente, mas poderia fazer agora, se fosse necessário.

Presta menos atenção ou tem menor conhecimento dos fatos do que antes.

Tem tanto conhecimento hoje quanto no passado.

Nunca prestou muita atenção nos eventos atuais e acharia muito difícil começar agora.

Nunca prestou muita atenção, mas poderia fazê-lo agora.

H. Presta atenção, entende e comenta novelas, jornais ou revistas?

Não lembra ou se confunde com o que viu ou leu.

Entende a ideia geral enquanto assiste ou lê, mas não se lembra

depois; não entende o assunto ou não tem opinião.

Menor atenção ou menos memória do que antes, maior dificuldade de entender a piada, o que antes era feito rapidamente. Entende tão rápido como antes.

Nunca prestou muita atenção ou comentou sobre a TV, nunca leu muito e provavelmente teria muita dificuldade de começar agora. Nunca leu ou assistiu muito à TV, mas poderia normalmente como

D. Joga baralho, xadrez, faz palavras cruzadas, trabalhos manuais

\begin{tabular}{c} 
ou tem algum outro passatempo? \\
\hline Fazia, mas atualmente não faz ou faz raramente e com
\end{tabular} qualquer pessoa, se tentasse. grande dificuldade. Precisa de ajuda frequente.

I. Lembra de compromissos, tarefas domésticas, eventos familiares (como aniversários) e medicações? Outra pessoa passou a fazer isso pelo idoso.

Tem que ser lembrado algumas vezes (mais do que antes ou mais do que a maioria das pessoas).

Realiza sem ajuda, porém com mais dificuldade do que antes. Realiza as atividades sem nenhuma ajuda.

Nunca fez e teria dificuldade para começar a fazer agora. Lembra sozinho, mas depende de lembretes, calendários ou agendas. Lembra dos compromissos tão bem como antes.

Nunca teve que se preocupar com compromissos, eventos familiares e medicações, e provavelmente teria muita dificuldade de começar agora.

Não fazia regularmente, mas poderia fazer agora, se fosse necessário.

Nunca teve de lidar com essas coisas no passado, mas poderia fazer isso normalmente como qualquer pessoa, se tentasse.

(continua) 


\begin{tabular}{|c|c|}
\hline E. Esquenta água, faz café ou chá e desliga o fogão? & $\begin{array}{l}\text { J. Sai do bairro, dirige, anda, pega ou troca de ônibus, trem ou } \\
\text { avião? }\end{array}$ \\
\hline $\begin{array}{l}\text { Atualmente alguém passou a fazer alguma ou todas essas atividades } \\
\text { pelo idoso. }\end{array}$ & $\begin{array}{l}\text { Atualmente não faz mais sozinho(a) ou alguém passou a acompanhá-lo } \\
\text { nessas atividades. }\end{array}$ \\
\hline Precisa de ajuda ou tem problemas frequentes. & Consegue se locomover pela vizinhança, mas se perde ao sair dela. \\
\hline Realiza as atividades sem ajuda, mas às vezes tem problema. & Tem mais problemas do que antes (p.ex.: às vezes se perde). \\
\hline Realiza as atividades sem nenhuma ajuda. & Desloca-se tão bem quanto antes. \\
\hline Nunca fez e teria dificuldade para começar a fazer agora. & $\begin{array}{l}\text { Raramente fazia essas atividades sozinho(a) e teria alguma dificuldade } \\
\text { de fazê-las agora. }\end{array}$ \\
\hline Não fazia regularmente, mas poderia fazer agora, se fosse necessário. & $\begin{array}{l}\text { Nunca teve que sair sozinho(a) muitas vezes no passado, mas poderia } \\
\text { fazer agora se fosse necessário. }\end{array}$ \\
\hline
\end{tabular}

Para avaliar potenciais correlações residuais, em todas as etapas analíticas foram utilizados os Índices de Modificação (IM), tendo em vista o ponto de corte > 10, a partir do qual é aceita a redundância de conteúdo em pares de itens 18. As análises estatísticas foram conduzidas no software Mplus 7.1 (http://www.statmodel.com/index.shtml).

\section{Resultados}

Os informantes tinham, em média, 43,2 anos de idade (DP = 14,6), eram majoritariamente do sexo feminino $(80,8 \%)$ e filhos dos idosos (46,5\%). Os idosos, por sua vez, tinham média de idade de 78 anos $(\mathrm{DP}=7,5), 72 \%$ eram do sexo feminino, $50,8 \%$ possuíam oito anos ou mais de estudo e $43,7 \%$ tinham renda pessoal mensal maior ou igual a 5,1 salários mínimos.

A Tabela 1 exibe a matriz de correlações policóricas para os dez itens do FAQ-BR.

Os resultados da AFC que avaliou a estrutura unidimensional do FAQ-BR evidenciaram que todos os itens se mostraram consistentes em dimensão única com altas cargas fatoriais. Os IM não indicaram a necessidade de explorar correlações residuais. Os índices de adequação de ajuste do modelo CFI e TLI mostraram-se adequados, mas o RMSEA, não (Tabela 2). Logo, a estrutura unidimensional do FAQ-BR proposta originalmente por Pfeffer et al. 7 não foi sustentada. Assim, soluções alternativas foram testadas.

Embora o limite superior do IC90\% do RMSEA tenha ficado acima do desejado, mas não o suficiente para sugerir um mau ajuste do modelo (valores $>0,10$ indicariam mau ajuste e consequente rejeição 15), os resultados da AFC/E evidenciaram uma solução bidimensional como a mais parcimoniosa para o FAQ-BR, com índices de ajuste incremental adequados e correlação entre as dimensões aceitável (Tabela 3). Os IM não indicaram a necessidade de explorar correlações residuais. Todos os itens apresentaram cargas fatoriais satisfatórias, sendo que os itens A, B, C, D, E, F e J compuseram uma dimensão que poderia ser nomeada Autonomia/Independência. Por outro lado, os itens G, H e I compuseram outra dimensão, que poderia corresponder a Memória/Cognição.

A estrutura bidimensional do FAQ-BR postulada na AFC/E foi reavaliada por AFC estrita, e foi corroborada como a mais parcimoniosa, sem cargas cruzadas e com índices de ajuste satisfatórios, exceto, de forma limítrofe, o limite superior do IC90\% do RMSEA. As cargas fatoriais mostraram-se altas, e os IM não indicaram a necessidade de explorar quaisquer correlações residuais. A correlação entre as dimensões indicou validade fatorial discriminante aceitável e de menor e, portanto, melhor valor que aquele encontrado na AFC/E prévia. A CC apresentou valores elevados e satisfatórios, evidenciando adequada consistência interna da escala (Tabela 4). 
Tabela 1

Matriz de correlações policóricas dos itens da versão brasileira do Functional Activities Questionnaire (FAQ-BR).

\begin{tabular}{|c|c|c|c|c|c|c|c|c|c|c|}
\hline & A & B & C & D & E & $\mathbf{F}$ & G & $\mathbf{H}$ & I & $\mathrm{J}$ \\
\hline A & 1,000 & & & & & & & & & \\
\hline B & 0,720 & 1,000 & & & & & & & & \\
\hline C & 0,787 & 0,661 & 1,000 & & & & & & & \\
\hline D & 0,653 & 0,535 & 0,680 & 1,000 & & & & & & \\
\hline E & 0,666 & 0,577 & 0,741 & 0,602 & 1,000 & & & & & \\
\hline $\mathbf{F}$ & 0,715 & 0,568 & 0,781 & 0,698 & 0,824 & 1,000 & & & & \\
\hline G & 0,677 & 0,591 & 0,663 & 0,618 & 0,626 & 0,611 & 1,000 & & & \\
\hline H & 0,675 & 0,571 & 0,655 & 0,594 & 0,624 & 0,617 & 0,794 & 1,000 & & \\
\hline 1 & 0,618 & 0,538 & 0,597 & 0,514 & 0,606 & 0,573 & 0,717 & 0,682 & 1,000 & \\
\hline J & 0,783 & 0,625 & 0,888 & 0,705 & 0,698 & 0,789 & 0,646 & 0,626 & 0,544 & 1,000 \\
\hline
\end{tabular}

Tabela 2

Análise fatorial confirmatória da estrutura unidimensional da versão brasileira do Functional Activities Questionnaire (FAQ-BR).

\begin{tabular}{lc}
\hline Itens & $\lambda$ \\
\hline A. Controla finanças & 0,858 \\
B. Gerencia documentos & 0,733 \\
C. Faz compras & 0,910 \\
D. Tem algum passatempo & 0,753 \\
E. Esquenta água, faz café/chá e desliga o fogão & 0,833 \\
F. Prepara uma refeição completa & 0,869 \\
G. Acompanha eventos atuais & 0,837 \\
H. Presta atenção, entende/comenta novela ou jornal & 0,814 \\
I. Lembra de compromissos, tarefas ou medicações & 0,733 \\
J. Sai do bairro, dirige ou usa transporte público & 0,903 \\
CFI & 0,974 \\
TLI & 0,967 \\
RMSEA (IC90\%) & $0,110(0,097-0,123)$ \\
\hline
\end{tabular}

$\lambda$ : cargas fatoriais; CFI: Comparative Fit Index; IC90\%: intervalo de 90\% de confiança; RMSEA: Root Mean Square Error of Aproximation; TLI: Tucker-Lewis Index.

\section{Discussão}

O principal objetivo deste estudo foi avaliar se a estrutura dimensional e a consistência interna do FAQ-BR - com única dimensão, conforme concebida por Pfeffer et al. 7 - captam adequadamente o constructo capacidade funcional do idoso em uma população distinta daquela onde foi originalmente aplicado. As evidências aqui apresentadas sugerem adequada consistência interna da escala, mas estrutura bidimensional.

A primeira dimensão incorporou atividades instrumentais de vida diária complexas, tais como “comprar roupas, utilidades domésticas e artigos de mercearia sozinho(a)" (item C) e "sair do bairro, dirigir, andar, pegar ou trocar de ônibus, trem ou avião” (item J), que apresentaram as maiores cargas fatoriais dessa dimensão. A outra, por sua vez, incorporou atividades instrumentais de vida diária 
Tabela 3

Análise fatorial confirmatória/exploratória da estrutura bidimensional da versão brasileira do Functional Activities Questionnaire (FAQ-BR).

\begin{tabular}{lcc}
\hline Itens & $\boldsymbol{\lambda}_{\mathbf{1}}$ & $\boldsymbol{\lambda}_{\mathbf{2}}$ \\
\hline A. Controla finanças & 0,600 & \\
B. Gerencia documentos & 0,449 & \\
C. Faz compras & 0,910 & \\
D. Tem algum passatempo & 0,594 & \\
E. Esquenta água, faz café/chá e desliga o fogão & 0,762 & 0,947 \\
F. Prepara uma refeição completa & 0,902 & 0,852 \\
G. Acompanha eventos atuais & & 0,752 \\
H. Presta atenção, entende/comenta novela ou jornal & & \\
I. Lembra de compromissos, tarefas ou medicações & & \\
J. Sai do bairro, dirige ou usa transporte público & 0,991 & \\
Correlação entre dimensões & 0,792 & \\
CFI & 0,990 & \\
TLI & 0,983 & \\
RMSEA (IC90\%) & $0,079(0,064-0,094)$ & \\
\hline
\end{tabular}

$\lambda_{1}$ e $\lambda_{2}$ : cargas fatoriais nas dimensões Autonomia/Independência e Memória/Cognição, respectivamente; CFI: Comparative Fit Index; IC90\%: intervalo de 90\% de confiança; RMSEA: Root Mean Square Error of Aproximation; TLI: Tucker-Lewis Index.

\section{Tabela 4}

Análise fatorial confirmatória da estrutura bidimensional da versão brasileira do Functional Activities Questionnaire (FAQ-BR).

\begin{tabular}{lcc}
\hline Itens & $\boldsymbol{\lambda}_{\mathbf{1}}$ & $\boldsymbol{\lambda}_{\mathbf{2}}$ \\
\hline A. Controla finanças & 0,872 & \\
B. Gerencia documentos & 0,744 & \\
C. Faz compras & 0,918 & \\
D. Tem algum passatempo & 0,766 & \\
E. Esquenta água, faz café/chá e desliga o fogão & 0,845 & \\
F. Prepara uma refeição completa & 0,878 & 0,896 \\
G. Acompanha eventos atuais & & 0,878 \\
H. Presta atenção, entende/comenta novela ou jornal & & 0,799 \\
I. Lembra de compromissos, tarefas ou medicações & & \\
J. Sai do bairro, dirige ou usa transporte público & 0,910 & 0,91 \\
Correlação entre dimensões & 0,654 & \\
CC & 0,90 & \\
CFI & 0,990 & \\
TLI & 0,987 & \\
RMSEA (IC90\%) & $0,070(0,056-0,083)$ &
\end{tabular}

$\lambda_{1}$ e $\lambda_{2}$ : cargas fatoriais nas dimensões Autonomia/Independência e Memória/Cognição, respectivamente; CC: confiabilidade composta; CFI: Comparative Fit Index; IC90\%: intervalo de 90\% de confiança; RMSEA: Root Mean Square Error of Aproximation; TLI: Tucker-Lewis Index. 
também consideradas complexas, mas com diferentes demandas processuais, relacionadas à cognição e à memória, tal como "acompanhar eventos atuais no bairro ou nacionalmente" (item G) e "prestar atenção, entender e comentar novelas, jornais ou revistas” (item H), que apresentaram as maiores cargas fatoriais dessa dimensão.

Altas cargas fatoriais $(\geq 0,845)$ foram identificadas nos itens A, C, E, F, G, que compuseram a dimensão Autonomia/Independência, e H e J, que compuseram a dimensão Memória/Cognição. No estudo que descreve o desenvolvimento do FAQ, Pfeffer et al. 7 sublinham que alguns itens, como os citados acima, possivelmente representam os níveis mais elevados de habilidades físicas e mentais necessárias para a manutenção de uma vida independente e autônoma na velhice.

Embora o FAQ tenha sido apresentado à comunidade científica há mais de trinta anos, foi pouco estudado e, possivelmente, encontra-se em fase de desenvolvimento, com espaço para avaliações adicionais. Na extensa revisão de literatura efetuada pelos autores, não foram identificados estudos de avaliação da estrutura dimensional do FAQ aplicado ao informante, o que impediu a comparação de nossos resultados. Sabe-se apenas que ele deriva de um modelo conceitual em dimensão única concebido por Pfeffer et al. 7, o que não foi corroborado pelo presente estudo.

O estudo de Assis et al. 10, tal como este, evidenciou bidimensionalidade para o FAQ, quando aplicado a 161 idosos não institucionalizados usuários de um serviço de saúde do Brasil. A despeito das limitações do método adotado para avaliação da estrutura dimensional dessa versão em português do FAQ - oriunda de um processo de adaptação transcultural desconhecido - e de alguns itens diferentes da escala original 7, os itens G, H e I também compuseram uma dimensão no estudo, ainda que o item I tenha apresentado cargas fatoriais cruzadas ${ }^{10}$. Assim, a escala parece atender à exigência mínima de três indicadores por dimensão, o que permite um mapeamento adequado do crescente de intensidade do conteúdo dimensional 19,20.

Diante disso, vale sublinhar que, embora o estudo de Assis et al. 10 tenha marcantes diferenças com o estudo em tela, especialmente no tocante à aplicação da escala entre idosos e não entre seus informantes, para quem foi originalmente proposta 7, nossos resultados corroboram as únicas evidências conhecidas até então sobre a estrutura (bi)dimensional do FAQ.

A correlação entre as dimensões do FAQ-BR (aqui nomeadas Autonomia/Independência e Memória/Cognição, por seus itens caracterizarem manifestações desses constructos) foi relativamente alta, mas não o suficiente para sugerir falta de validade fatorial discriminante 15 . Diante desse resultado, cabe destacar que a capacidade funcional do idoso interage de tal forma com a dimensão cognitiva que uma alteração nessa dimensão implica algum prejuízo na independência e autonomia, comprometendo, assim, seu desempenho em atividades de vida diária 4,21. Provavelmente, essa simbiose é o motivo de a correlação entre as dimensões do FAQ-BR ter se mostrado alta, mas, ainda assim, capaz de diferenciar um constructo de outro ${ }^{15}$. Desse modo, os dez itens da escala mostraram-se capazes de capturar diferentes aspectos da capacidade funcional do idoso, quais sejam: Autonomia/Independência 1,2 e Memória/Cognição 4,21.

A consistência interna do FAQ-BR foi considerada satisfatória, sugerindo que todos os itens representam de modo coerente o mesmo constructo latente: a capacidade funcional do idoso. Segundo seu breve histórico psicométrico, o FAQ apresenta apropriada consistência interna, pois estudos conduzidos entre informantes de idosos checos 22 e norte-americanos 23 encontraram coeficientes alfa de Cronbach de 0,79 e 0,90, respectivamente. Em investigação anterior, também foi estimada satisfatória consistência interna da escala em nossa população de estudo (alfa de Cronbach = 0,95) 12

O presente estudo requer interpretação à luz de suas limitações e potencialidades. Como limitação, assinala-se que os modelos foram testados com um único conjunto de dados, carecendo ainda de estudos de replicação e confirmação. Como pontos fortes, destacam-se o tamanho amostral apropriado para conferir precisão às estimativas e o uso de técnicas estatísticas adequadas para estudos de validação de escalas de aferição. Além disso, vale sublinhar que este é o primeiro estudo a avaliar a estrutura dimensional do FAQ com base no relato do informante. Um aspecto que também merece destaque refere-se à operacionalização do FAQ-BR por telefonema, diferente da abordagem do estudo original em que a escala foi aplicada via entrevista face a face ${ }^{7}$. Com essa operacionalização, foi possível constatar a praticidade de aplicação dessa escala por entrevistadores treinados em tempo breve.

É recomendável que estudos futuros avaliem, em uma perspectiva comparativa, o comportamento do FAQ-BR entre idosos e seus informantes, já que tal avaliação não é possível no FIBRA-RJ, pois a 
escala foi aplicada somente aos informantes. Desse modo, seria descoberta a aplicabilidade da escala em situações em que o idoso fosse capaz de respondê-la. Seria também interessante avaliar a validade preditiva da capacidade funcional do idoso em relação a diferentes desfechos de saúde, como a demência, o que seria uma proposta viável no contexto de estudos longitudinais, como o FIBRA-RJ. No conjunto, essas avaliações complementariam o escrutínio das propriedades psicométricas do FAQ-BR, no sentido de reforçar a adequabilidade de seu uso no Brasil.

Em suma, a estrutura bidimensional de melhor ajuste para o FAQ-BR apresentou cargas fatoriais altas para todos os itens e índices de ajuste do modelo adequados, além de satisfatória consistência interna e aceitável validade fatorial discriminante. Esses achados são importantes, já que revelam a capacidade de a escala capturar o construto capacidade funcional do idoso em um contexto sociocultural distinto daquele em que foi desenvolvido. Assim, o FAQ-BR pode ser considerado um instrumento bidimensional apropriado para aferir a capacidade funcional do idoso, com base no relato do informante. Portanto, seu uso em pesquisas com populações semelhantes é recomendado, seja considerando a escala em sua forma bruta por meio de escores totais formados pelo somatório dos escores de itens componentes, seja como variáveis latentes por meio de escores fatoriais obtidos via análises de modelos de equação estrutural.

\section{Colaboradores}

R. T. Jomar concebeu e delineou o estudo, conduziu a análise de dados, redigiu e aprovou a versão final do artigo. R. A. Lourenço e C. S. Lopes conceberam o estudo, revisaram e aprovaram a versão final do artigo.

\section{Referências}

1. Veras RP. Envelhecimento populacional contemporâneo: demandas, desafios e inovações. Rev Saúde Pública 2009; 43:548-54.

2. Lima-Costa MF, Facchini LA, Matos DL, Macinko J. Mudanças em dez anos das desigualdades sociais em saúde dos idosos brasileiros (1998-2008). Rev Saúde Pública 2012; 46 Suppl 1:100-7.

3. Alves LC, Leite IC, Machado CJ. Conceituando e mensurando a incapacidade funcional da população idosa: uma revisão de literatura. Ciênc Saúde Coletiva 2008; 13:1199-207.

4. Paixão Jr. CM, Reichenheim ME. Uma revisão sobre instrumentos de avaliação do estado funcional do idoso. Cad Saúde Pública 2005; 21:7-19.

5. Davis LL. Assessing functional ability in persons with dementia: using family as informants. J Neurosci Nurs 2001; 33:194-202. 
6. Jorm AF, Broe GA, Creasey H, Sulway MR, Dent O, Fairley MJ, et al. Further data on the validity of the Informant Questionnaire on Cognitive Decline in the Elderly (IQCODE). Int J Ger Psychiatry 1996; 11:131-9.

7. Pfeffer RI, Kurosaki TT, Harrah Jr. CH, Chance JM, Filos S. Measurement of functional activities in older adults in the community. J Gerontol 1982; 37:323-9.

8. Assis LO, Assis MG, de Paula JJ, Malloy-Diniz LF. O Questionário de Atividades Funcionais de Pfeffer: revisão integrativa da literatura brasileira. Estud Interdiscip Envelhec 2015, 20:297-324.

9. Vasconcelos LG, Brucki SMD, Bueno OFA. Cognitive and functional dementia assesment tools. Review of Brazilian literature. Dement Neuropsychol 2007; 1:18-23.

10. Assis LO, de Paula JJ, Assis MG, Moraes EM, Malloy-Diniz LF. Psychometric properties of the Brazilian version of Pfeffer's Functional Activities Questionnaire. Front Aging Neurosci $2014 ; 6: 255$.

11. Herdman M, Fox-Rushby J, Badia X. A model of equivalence in the cultural adaptation of HRQoL instruments: the universalist approach. Qual Life Res 1998; 7:323-35.

12. Sanchez MAS, Correa PCR, Lourenço RA. Cross-cultural adaptation of the "Functional Activities Questionnaire - FAQ" for use in Brazil. Dement Neuropsychol 2011; 5:322-7.

13. Lourenço RA, Sanchez MA, Moreira VG, Ribeiro PCC, Perez M, Campos GC, et al. Fragilidade em Idosos Brasileiros - FIBRA-RJ: metodologia de pesquisa dos estudos de fragilidade, distúrbios cognitivos e sarcopenia. Revista Hospital Universitário Pedro Ernesto 2015; 14:13-23.

14. Lourenço RA, Veras RP. Mini-Exame do Estado Mental: características psicométricas em idosos ambulatoriais. Rev Saúde Pública 2006; 40:712-9.
15. Brown TA. Confirmatory factor analysis for applied research. 2nd Ed. New York: The Guilford Press; 2015.

16. Hair JF, Black WC, Babin BJ, Anderson RE, Tatham RL. Análise multivariada de dados. 6a Ed. Porto Alegre: Bookman; 2009.

17. Loehlin JC. Latent variable models: an introduction to factor, path, and structural equation analysis. 4th Ed. Mahwah: Lawrence Erlbaum; 2003.

18. Reichenheim ME, Hökerberg YHM, Moraes CL. Assessing construct structural validity of epidemiological measurement tools: a seven-step roadmap. Cad Saúde Pública 2014; 30:927-39.

19. Velicer WF, Fava JL. Effects of variable and subject sampling on factor pattern recovery. Psychol Methods 1998; 3:231-51.

20. Pett MA, Lackey NR, Sullivan JJ. Making sense of factor analysis: the use of factor analysis for instrument development in health care research. London: Sage Publications; 2003.

21. Chaves ML, Godinho CC, Porto CS, Mansur L, Carthery-Goulart MT, Yassuda MS, et al. Cognitive, functional and behavioral assessment: Alzheimer's disease. Dement Neuropsychol 2011; 5:153-66.

22. Bezdícek O, Lukavský J, Preiss M. Functional Activities Questionnaire, Czech version - a validation study. Cesk Neurol Neurochir 2011; 74:36-42.

23. Tappen RM, Rosselli M, Engstrom G. Evaluation of the Functional Activities Questionnaire (FAQ) in cognitive screening across four American ethnic groups. Clin Neuropsychol 2010; 24:646-61. 


\section{Abstract}

This study aimed to assess the dimensional structure and internal consistency of the Brazilian version of the Functional Activities Questionnaire (FAQ-BR) for measurement of functional capacity in the elderly, based on the informant's report. This was a cross-sectional study with 525 noninstitutionalized elderly informants, in which confirmatory factor analyses (CFA) and exploratory/ confirmatory factor analyses (E/CFA) modeled according to CFA principles were used to identify the most parsimonious model and that with the best fit. The internal consistency of the FAQ-BR was assessed by composite reliability, and correlations between its dimensions were examined to investigate discriminant factor validity. CFA did not corroborate the unidimensional structure as originally proposed. Next, E/CFA fit a bidimensional structure that was reassessed by CFA, displaying a model without cross loads as the most parsimonious and with adequate fit indices. The internal consistency of FAQ-BR was considered satisfacto$r y$, and the correlation between its dimensions was acceptable. These findings are important, since they demonstrate the scale's capacity to capture the elderly's functional capacity construct in a different sociocultural context from that in which it was originally developed. The FAQ-BR can thus be considered an appropriate bidimensional instrument for measuring the elderly's functional capacity based on the informant's report, and its use in studies with similar populations is recommended.

Evaluation of Research Programs and Tools; Factor Analysis, Statistical; Validation Studies; Activities of Daily Living; Health of the Elderly

\section{Resumen}

El objetivo de este artículo fue evaluar la estructura dimensional y la consistencia interna de la versión brasileña del Functional Activities Questionnaire (FAQ-BR) para la valoración de la capacidad funcional del anciano, en base al relato del informante. Se trata de un estudio seccional, desarrollado con 525 informantes de ancianos no institucionalizados, donde los análisis factoriales confirmatorios (AFC) y análisis factoriales exploratorios $(A F C / E)$, fueron modelados según los principios de la AFC, así como usados para identificar el modelo más parsimonioso y más bien ajustado. La consistencia interna del FAQ-BR se evaluó por la fiabilidad compuesta, y se examinaron las correlaciones entre sus dimensiones, a fin de investigar la validez factorial discriminante. La AFC no corroboró la estructura unidimensional originalmente propuesta. En seguida, la AFC/E ajustó una estructura bidimensional que se reevaluó por parte de la AFC, evidenciando un modelo sin cargas cruzadas como el más parsimonioso y con adecuados indices de ajuste. La consistencia interna del FAQ-BR se consideró satisfactoria, y la correlación entre sus dimensiones, aceptable. Estos hallazgos son importantes, pues revelan la capacidad de la escala de aprehender el constructo capacidad funcional del anciano en un contexto sociocultural distinto de aquel en el que se desarrolló. Por ello, el FAQ-BR puede ser considerado un instrumento bidimensional, apropiado para evaluar la capacidad funcional anciano, basándose en el relato del informante, y se recomienda su uso en investigaciones con poblaciones semejantes.

Evaluación de Programas e Instrumentos de Investigación; Análisis Factorial; Estudios de Validación; Actividades Cotidianas; Salud del Anciano
Recebido em 09/Dez/2017

Versão final reapresentada em 16/Mar/2018

Aprovado em 18/Mai/2018 